118 TRANSFORMING MDT WORKING IN PALLIATIVE AND END OF LIFE CARE ACROSS ACUTE AND COMMUNITY SETTINGS

Sara Morley, Catherine Hardie, Sindy Dhallu. Walsall Healthcare NHS Trust

10.1136/bmjspcare-2018-ASPabstracts. 145

As a forward thinking Integrated specialist Palliative and End of Life Care team working across community and acute settings within Walsall we are constantly appraising our service and looking to improve how we deliver the best possible care to our patients and carers.

Having an existing Palliative and End of Life Care MDT we wanted to refine the process and develop a more accessible platform for non specialist colleagues in a supportive environment to encourage open conversations with a more truly integrated pathway.

This process has involved the engagement of partner organisations such as our local Hospice St Giles Hospice Walsall, acute based teams, community specialist palliative care, GP's community matrons and district nursing teams, specialist services such as COPD HF, learning disabilities and mental health.

In the last couple of years the SPC has been critically appraised by its members and over the last twelve months we have trialled a rotational chair in order to promote the skills of clinicians outside the medical framework by introducing an innovative approach to the chairing of the MDT.

We also then focused on rotational locations for the meeting, a different time slot and the introduction of bringing cases for reflection. These focuses have promoted staff development, leadership skills, accessibility: bringing everyone to the table, revalidating interventions.

However most significantly the changes have increased engagement with attendee numbers rising significantly.A Survey Monkey demonstrate the value placed upon the SPC MDT both inside and outside the historical circles of palliative and end of life care.

This audit serves to appraise how these changes have affected the SPC creating a platform for further appraisal and improvement.

\section{A RETROSPECTIVE AUDIT OF THE PALLIATIVE CARE TEAM ELECTRONIC MDT PROFORMA AT THE ROYAL MARSDEN NHS FOUNDATION TRUST}

Geoffrey Wells, Angela Halley, Carol Marlow, Joanne Droney. The Royal Marsden Hospital

\subsection{6/bmjspcare-2018-ASPabstracts. 146}

Background The Palliative Care (PC) Multidisciplinary Team $(\mathrm{MDT})$ in a tertiary referral oncology hospital meets weekly across two sites. All new referrals and all inpatients under the PC team are discussed. In June 2016 an electronic MDT (eMDT) proforma was developed to record data including performance status, phase of illness, estimated prognosis, spiritual, psychological and welfare needs and Urgent Care Planning. A weekly MDT register is taken to establish core membership attendance and adherence to recommendations in the National Cancer Peer Review Programme (2012). A retrospective audit of the data was conducted.

Methods Weekly eMDT data (1 st June 2016 - 31 st March 2017) and PC inpatient lists were reviewed. All patient discussions were retrospectively analysed to determine whether we were achieving departmental standards. Microsoft Excel was used for statistical analysis. An audit standard of 75\% was set for core MDT attendance and for recording all data.

Results 803 individual patient discussions took place. $75 \%$ core attendance was achieved. Psychological needs were documented on the e-MDT form in only $38.5 \%$ and PPD in $25.7 \%$ overall. $67(8.3 \%)$ of patients had an initial prognosis of 'hours to days' when first discussed. Of these 54 (81\%) had a documented preferred place of care (PPC) and 52 (78\%) had a documented preferred place of death (PPD).

Conclusions Electronic documentation of palliative care MDT discussions and decisions facilitates analysis and quality improvement. On the basis of this audit the operational policy has been updated. The psychology team have been invited to join the weekly MDT. The audit standards regarding PPD have been amended to only include patients who are identified as dying.

\section{DEFINING AND MANAGING THE COMPLEX PATIENT: STAFF PERCEPTIONS IN COMMUNITY SPECIALIST PALLIATIVE CARE}

Jonathan Pickard. Northumbria Healthcare NHS Foundation Trust

\subsection{6/bmjspcare-2018-ASPabstracts. 147}

Aim To evaluate factors perceived as contributing to patient complexity in a community specialist palliative care team (SPCT) and assess confidence of community staff in identifying and managing such patients.

Background Multiple co-morbidities, increased healthcare usage and psychosocial vulnerabilities feature broadly in descriptions of complexity and are prevalent within the palliative care demographic. Shared awareness of more-complex patients is necessary for cohesive care, effective handover and learning. Despite this, it is unclear how confidently SPCTs identify and manage such patients.

Methods 14 nurses, HCAs and AHPs in a mixed-experience community SPCT in North East England were surveyed. Participants scored different biopsychosocial-spiritual aspects on a scale of 1 to 5 according to perceived contribution to complexity $(1=$ weak and $5=$ strong contribution). Scores for each aspect were totaled. Participants then self-rated agreement with statements around complex patient identification, management and team support. Percentages of respondents self-rating $>6 / 10$ were calculated.

Results $23.7 \%$ of patients were rated more complex than average. Exhausting clinical management options and high symptom burden contributed most to complexity (aggregated score 65/70), followed by rapid condition changes (64/70) and requirement for frequent interventions/admissions (63/70). $57.1 \%$ of staff felt at least somewhat confident in identifying and managing complex patients, with only $35.7 \%$ aware of robust systems for flagging complex individuals. $78.6 \%$ agreed a formalised system (e.g. 'Virtual Ward') could improve complex patient care.

Discussion Self-perceived influencers of complexity are congruent with the literature, yet confidence in identifying and managing such patients is low. Subgroup analysis shows that although more-experienced staff have greater confidence in managing complexity, they perceive fewer opportunities to flag and discuss complex individuals, highlighting the need for a cross-sectional approach to enhancing complex patient care. 